schedule (e.g., frequency of night shifts, duration in years) are needed. Further, research to the (mediating) role of lifestyle behaviours in the health effects of shift work is recommended, as this may offer potential for preventive strategies.

\section{7e WORKLOAD AND EARLY FUNCTIONAL AGEING AMONG HOSPITAL WORKERS}

${ }^{1}$ Maria Carmen Martinez, ${ }^{2}$ Maria do Rosário Dias de Oliveira Latorre, ${ }^{3}$ Frida Marina Fischer. 'Independent consultant in Epidemiology in Occupational Health. WAF Informática, São Paulo, Brazil; ${ }^{2}$ Department of Epidemiology, School of Public Health, University of São Paulo, Brazil; ${ }^{3}$ Department of Environmental Health, School of Public Health, University of São Paulo, Brazil

\subsection{6/oemed-2018-ICOHabstracts. 104}

Introduction Early functional ageing - EFA is the progressive work ability - WA impairment preceding the chronological ageing. Hospital work is characterised by physical and mental workloads arising from work stressors that can influence functional ageing. The aim of this follow-up study was to assess the association between psychosocial work stressors and functional ageing in a group of Brazilian hospital employees.

Methods This is a 3 year follow-up (2009-2012) study of 423 hospital workers of São Paulo, Brazil. We included only workers with excellent/good WA at the baseline. At baseline workers filled a form on sociodemographic, lifestyle, and occupational questions including the Brazilian versions of Job Stress Scale, Effort-Reward Questionnaire, Work-Related Activities That May Contribute To Job-Related Pain and/or Injury, and Work Ability Index. Moderate/poor WA were considered as EFA. Changes from excellent/good to moderate/poor WA were the dependent variable. We used logistic regression models adjusted for potential confounders.

Results The average age was 36.0 y $(\mathrm{SD}=8.3), 30.7 \%$ were over $40 \mathrm{y}, 72.1 \%$ were females. At the end of follow-up the exposure to work stressors had worsened: job strain (20.3\%), social support (22.7\%), effort-reward imbalance (18.7\%), overcommitment (18.4\%) and Work-Related Activities That May Contribute To Job-Related Pain and/or Injury (13.9\%). Eighteen percent of the workers shifted to moderate or poor WA. High levels of exposure to psychosocial work stressors were significantly associated with decreased work ability: job strain $(\mathrm{OR}=2.81)$ and effort-reward imbalance $(\mathrm{OR}=3.21)$.

Discussion Work stressors were risk factors for work ability. The results showed the need for interventions to maintain hospital employees' work ability. Such strategies have implications for institutional and social policies to prevent early functional ageing.

\section{$1617 f$ COPING WITH SHIFTWORK: INDIVIDUAL AND TEAM STRATEGIES FOR HEALTH AND SAFETY}

1) Dorrian*, ${ }^{1} S$ Centofanti, ${ }^{2} \mathrm{~A}$ Colella, ${ }^{2} \mathrm{~L}$ Devine, ${ }^{2} \mathrm{C}$ Dingle, ${ }^{2} \mathrm{H}$ Galindo, ${ }^{2} \mathrm{~S}$ Pantelios, ${ }^{2} \mathrm{G}$ Brkic, ${ }^{3} \mathrm{C}$ Bull, ${ }^{3} \mathrm{~T}$ Almond, ${ }^{3} \mathrm{~V}$ Dhillon, ${ }^{1} \mathrm{MA}$ Carskadon, ${ }^{1} \mathrm{~S}$ Banks. ${ }^{1}$ University of South Australia, Adelaide, Australia; ${ }^{2}$ SA Health, Adelaide, Australia; ${ }^{3}$ CSIRO, Adelaide, Australia

\subsection{6/oemed-2018-ICOHabstracts. 105}

Introduction Shiftwork can negatively impact on performance, safety and health. Importantly, while some shiftworkers are profoundly affected, others cope very well. The aim of this project was to identify healthy shiftworkers (across measures of physical, behavioural and psychosocial health) in a group of Australian nurses and midwives, and to examine factors that may contribute to health across working life.

Methods This was a mixed methods study, combining survey, interview and biological measurements. Participating hospital nurses and midwives $(\mathrm{n}=96, \mathrm{f}=85$, age $=44.3 \pm 12.6 \mathrm{y}$, shiftwork experience $=18.9 \pm 12.7$ y) completed the Standard Shiftwork Index, which includes measures of health, social, domestic and work disruption, and coping styles. Participants could also participate in an interview $(n=22)$, and/or provide a saliva sample $(n=45)$, to measure telomere length (if our DNA were shoelaces, telomeres would be the aglets).

Results Cluster analysis revealed a 'Healthier' and a 'Less Healthy' cluster, with significantly worse scores across all health variables $(\mathrm{p}<0.05)$. Controlling for gender, age and work hours, the odds of being 'Less Healthy' were significantly $(p<0.05)$ : reduced for experienced shiftworkers $(\geq 20 y$ versus $<20 \mathrm{y}, \mathrm{OR}=0.16$ ); reduced by engaged coping style $(\mathrm{OR}=0.92)$; and increased by disengaged coping style $(\mathrm{OR}=1.20)$. Strategies for coping were aimed at promoting wakefulness/sleep at biologically difficult times, sustaining safe performance at work and while driving, and maintaining healthy diet, exercise and relationships. Strategies included the use of stimulants and sedatives, changes in composition/timing of food intake, changes in timing/distribution of sleep, advances/delays in undertaking safety-critical activities, and contributing to a culture of social support. Correlations between age and telomere length were negative in less experienced $(<20 \mathrm{y}$, $r=-0.22)$ and positive in more experienced $(\geq 20 \mathrm{y}, r=0.85)$ shiftworkers.

Conclusion Findings provided evidence of healthy ageing in experienced shiftworkers that was linked to coping style. Studying those who cope well provides an evidence-base for teaching successful behavioural strategies to improve worker health and safety.

\section{$1617 \mathrm{~g}$ ASSOCIATION OF INSOMNIA AND FATIGUE DUE TO SHIFT WORK IN MIDLIFE AND MOBILITY LIMITATIONS OVER 28 YEARS OF FOLLOW-UP}

${ }^{1,2} \mathrm{KC}$ Prakash*, ${ }^{1,2}$ Subas Neupane, ${ }^{3}$ Päivi Leino-Arjas, ${ }^{3}$ Mikko Härmä, ${ }^{1,2}$ ClasHåkan Nygård. ${ }^{1}$ Faculty of Social Sciences, Health Sciences, University of Tampere, Tampere, Finland; ${ }^{2}$ Gerontology Research Centre, University of Tampere, Tampere, Finland; ${ }^{3}$ Finnish Institute of Occupational Health, Helsinki, Finland

\subsection{6/oemed-2018-ICOHabstracts. 106}

Objectives We aimed to investigate the long-term association of insomnia and fatigue due to shift work (with and without night shifts) in midlife and mobility limitations (ML) among initially middle-aged subjects followed over 28 years.

Methods The Finnish Longitudinal Study on Ageing Municipal Employees (FLAME) was conducted by the Finnish Institute of Occupational Health from 1981 to 2009 among 6257 municipal workers. This study is based on the latest available response on ML questionnaires either from 1992 or 1997 or in $2009(n=4704)$. International Classification of Functioning (ICF) was used to code the nine mobility tasks included in the ML. Insomnia and fatigue due to shift work were assessed using the yes/no questions in the baseline. The Incidence Rate Ratio (IRR) and 95\% Confidence Interval (CI) for the association of insomnia and fatigue due to shift work and ML were assessed by using mixed Poisson regression. The results are presented separately for women and men in shift work with and without night shifts. 
Results After adjusting for age, smoking, alcohol use, BMI, physical exercise and chronic diseases, women engaged in shift work without night shifts in midlife had on average a $24 \%$ (due to shift work insomnia) and a $16 \%$ (due to shift work fatigue) higher risk for a unit increase in ML in old age than those without shiftwork insomnia and fatigue. Likewise, men engaged in shift work with night shifts in midlife had on average a $61 \%$ (insomnia) and a 66\% (fatigue) higher risk for a unit increase in ML in old age. Furthermore, women in shift work with night shifts and men in shift work without night shifts had on average a higher risk of ML, but the risk was attenuated and remained insignificant after adjustment.

Conclusions The findings of this prospective 28 year cohort study suggest that shift work related insomnia and fatigue in midlife have inverse effects on mobility functions in old age irrespective of gender and type of shift work, and indicates in the initiation of prevention of mobility decline in working life.

\section{EPIDEMIOLOGICAL STUDY BETWEEN TYPE 2 DIABETES AND BONE MINERAL DENSITY}

Kazuhiro Hidaka, Yoshiki Kuroda. Department of Public Health, Faculty of Medicine, University of Miyazaki

\subsection{6/oemed-2018-ICOHabstracts. 107}

Introduction In Japan, many people over 65 years old are working now. However, elderly working people frequently face to getting lifestyle diseases, so it would be important to control lifestyle diseases. Among lifestyle diseases, diabetes mellitus (DM) is a popular in the world. On the other hand, Osteoporosis is also an important disease for elderly workers, because osteoporosis is a risk to get fracture. There are some reports indicated that fractures was frequently in workers with DM. However, it was also reported that bone mineral density (BMD) in type $2 \mathrm{DM}$ patients was higher than non-diabetic persons. These reports were contradictory. Therefore, we planned to evaluate fracture risk and BMD of persons with type 2 DM comparing with healthy subjects.

Material and method We recruited 183 persons with type 2 DM (detail: 101 males, 82 females) under informed consent. We excluded persons with type 1 diabetes, rheumatic diseases. We collected individual data (age, sex, BMI, fasting blood sugar, average fasting blood sugar, $\mathrm{HbA1c}$ value, post history of fracture) and measure BMD using ultrasonic bone mass measurement.

Result

- BMD decreased with age. And BMD was higher than healthy subjects (Male and Female).

- In male, there was a significant positive correlation between MBD and BMI.

- In female, a significant positive correlation was found between BMD and fasting blood sugar.

- We didn't detect the difference concerning to fracture history between persons with DM and healthy persons.

Discussion We indicated BMD of DM persons was higher than healthy subjects. In addition, BMD of female was a significant positive correlation with the fasting blood sugar. But we could not indicate higher fracture risk in DM persons. We are planning to check whether fracture frequently happened or not in persons with DM.

\section{THE DETERIORATION TENDENCY OF HEALTH CONDITION IN AGE GROUP AND SEX BY FOLLOW-UP DATA}

${ }^{1} \mathrm{Y}$ Higuchi*, ${ }^{2} \mathrm{M}$ Ohta, ${ }^{3} \mathrm{M}$ Kumashiro. ${ }^{1}$ University of Teacher Education Fukuoka, Munakata, Japan; ${ }^{2}$ Fukuoka Women's University, Fukuoka, Japan; ${ }^{3}$ The Association for Preventive Medicine of Japan, Tokyo, Japan

\subsection{6/oemed-2018-ICOHabstracts. 108}

Introduction Along with the ageing society as a whole, the upper limit of the employment period in law has reached the age of 65 in Japan. It is thought that health risk increases in the elderly. In Japan, the age group subject to focused health promotion activities is considered to be in the $40 \mathrm{~s}$ and over. However, from the viewpoint of primary prevention, it is considered that measures to prevent disease are necessary before deterioration of health. Therefore, in order to obtain evidence on the deterioration of health condition, we have observed worker's health examination data over time.

Methods Health examination data for 14 years (2002-2015) were analysed. Within this period, the rate at which abnormal values appeared in blood pressure, GOT, total cholesterol, triglyceride, fasting blood glucose was calculated. The sample size is $n=25146$ who did not have missing values in all data. Results At the time of baseline (in 2002), abnormal values were found in the examined items, 55\% for males and 36\% for females. In 2015, they were 69\% (14\% increase) and $57 \%$ (21\% increase), respectively. By age group and gender, increase rate for male increased by $16 \%$ (38\% to $54 \%$ ) in their $20 \mathrm{~s}, 17 \%$ increase $(51 \%$ to $68 \%)$ in their $30 \mathrm{~s}, 9 \%$ increase in their $40 \mathrm{~s}(67 \%$ to $76 \%)$ and increased by $8 \%$ $(72 \%$ to $80 \%)$ in their $50 \mathrm{~s}$. For female, these rate increased by $4 \%$ (33\% to $37 \%)$, $19 \%$ (33\% to $52 \%), 32 \%(40 \%$ to $72 \%)$ and $11 \%(65 \%$ to $76 \%)$, respectively.

Conclusion With age, the appearance rate of abnormal values in major medical examination items increases. The tendency of the rate of increase varies depending on age group and. The rate of increase in male in their $20 \mathrm{~s}$ and $30 \mathrm{~s}$ is large, while in females the rate in their $40 \mathrm{~s}$ is large. From the viewpoint of disease prevention, it is necessary to develop health education to improve self-management ability and to review lifestyle habits from the stage before deterioration of health condition.

\section{OCCUPATIONAL SOCIAL CLASS AND SELF RATED HEALTH. A CROSS SECTIONAL STUDY OF OLDER IRISH ADULTS FROM THE IRISH LONGITUDINAL STUDY ON AGEING}

${ }^{1} \mathrm{~S}$ Kelly ${ }^{*},{ }^{2} \mathrm{~A}$ McCarren, ${ }^{3} \mathrm{~A}$ Reid. ${ }^{1}$ Specialist Registrar in Occupational Medicine, Tallaght Hospital, Dublin, Ireland; ${ }^{2}$ Dublin City University, Dublin, Ireland; ${ }^{3}$ Consultant Occupational Physician, Tallaght Hospital and Coombe Women and Infants University Hospital, Dublin, Ireland

\subsection{6/oemed-2018-ICOHabstracts.109}

Introduction Self-rated health has been shown to be an important predictor of future morbidity and mortality. We investigated the association between self-rated health and occupational social class in a population at work aged $\geq 50$ years in Ireland, and determined its relationship with demographic and health-related variables. 